\title{
A case of a metastatic SDHA mutated paraganglioma re-presenting twenty- three years after initial surgery
}

\section{Dear Editor,}

We have read with great interest the recent article by Tufton and coworkers reporting on the risk of metastasis in patients with paraganglioma (PGL) tumours associated with germline SDHA mutations (Tufton et al. 2017). Herein, we report a further case of a malignant PGL in a 46-year-old man with a succinate dehydrogenase complex flavoprotein subunit A (SDHA) germline mutation (c.91C > T, p.Arg31*). In the case we describe, following the initial surgical removal of a left-sided retroperitoneal PGL, twenty-three years elapsed before the development of a bony metastasis in the eighth left rib. This observation is similar to that of Tufton and coworkers who reported two patients, who developed metastatic disease in 16 and 37 years, respectively, following initial diagnosis (Tufton et al. 2017). During investigations for this case, we found that the rib metastasis was avid on 18-fluorodeoxyglucose (FDG) positron emission tomography (PET) computed tomography (CT), but not avid on radiolabeled I131 metaiodobenzylguanidine (MIBG) imaging (Fig. 1). This observation of SDHA-related malignant PGL is in keeping with SDHB- and SDHD-associated PGL, in which reduced avidity on MIBG imaging is due to reduced tumoural expression of noradrenaline transporters (Timmers et al. 2007). This clinical report supports the recent literature that suggests a risk of malignancy in patients with SDHA mutated PGL (Bausch et al. 2017, Casey et al. 2017, Tufton et al. 2017). The prolonged time interval between diagnosis and development of metastases in our case suggests that SDHA-related tumours are slow growing but it does support long-term surveillance programmes for patients with germline $S D H A$ mutations.

Although mutations in $S D H B, S D H C$ and $S D H D$ gene subunits were first identified as predisposition genes for the development of phaeochromocytoma (PC) and PGL tumours over 15 years ago, SDHA was first associated with PGL only seven years ago (Burnichon et al. 2010) and, more recently, with wild-type gastrointestinal stromal tumours (WT GIST) (Evenepoel et al. 2015). It is now recognised that the SDHA gene is the most common SDHx germline mutation implicated in development of SDH-deficient WT GIST (Boikos et al. 2016). Recently, the European-AmericanAsian Pheochromocytoma-Paraganglioma Registry Study Group reported on 34 index cases with germline SDHA mutations and PC or PGL tumours. This group described a high prevalence of head and neck PGL in the SDHA cohort $15 / 34(44 \%)$ and metastatic disease was reported in $4 / 34$ (12\%) (Bausch et al. 2017).

Here, we report the case of a 46-year-old man who first presented at age 23 years with headache, heat intolerance and abdominal pain. He was subsequently diagnosed with a left-sided retroperitoneal paragangliomama, which was surgically resected. The man had no family history of endocrine tumours and no additional relevant medical history. Following surgery, the patient was surveyed in primary care with annual urinary metanephrine testing. Twenty-three years later he was referred to our specialist neuroendocrine tumour service at Cambridge University Hospital NHS Foundation Trust, due to an elevated urinary normetanpehrine level (urinary normetanephrine $5870 \mathrm{nmol} / 24 \mathrm{~h}$, reference range 0-4900; urinary metanephrine $756 \mathrm{nmol} / 24 \mathrm{~h}$, reference range 0-2000), which was first observed 3 months earlier. On review, the patient denied symptoms suggestive of catecholamine excess and, importantly, denied any of the symptoms that he reported at the time of his initial presentation. Review of systems revealed that his only complaint was left side rib pain. Previous investigations in primary care included a plain chest radiograph which did not reveal any abnormality.

We performed plasma metanephrine testing and found an elevated normetanephrine level $(2864 \mathrm{pmol} / \mathrm{L}$, reference range $<1000 \mathrm{pmol} / \mathrm{L}$ ) in the context of normal plasma metanephrine $(197 \mathrm{pmol} / \mathrm{L}$, reference range $<600 \mathrm{pmol} / \mathrm{L})$ and methoxytyramine measurements $(95.6 \mathrm{pmol} / \mathrm{L}$, reference range $<180 \mathrm{pmol} / \mathrm{L}$ ). In light of the elevated normetanephrine level in urine and plasma, CT of the

This work is licensed under a Creative Commons Attribution 3.0 Unported License. 


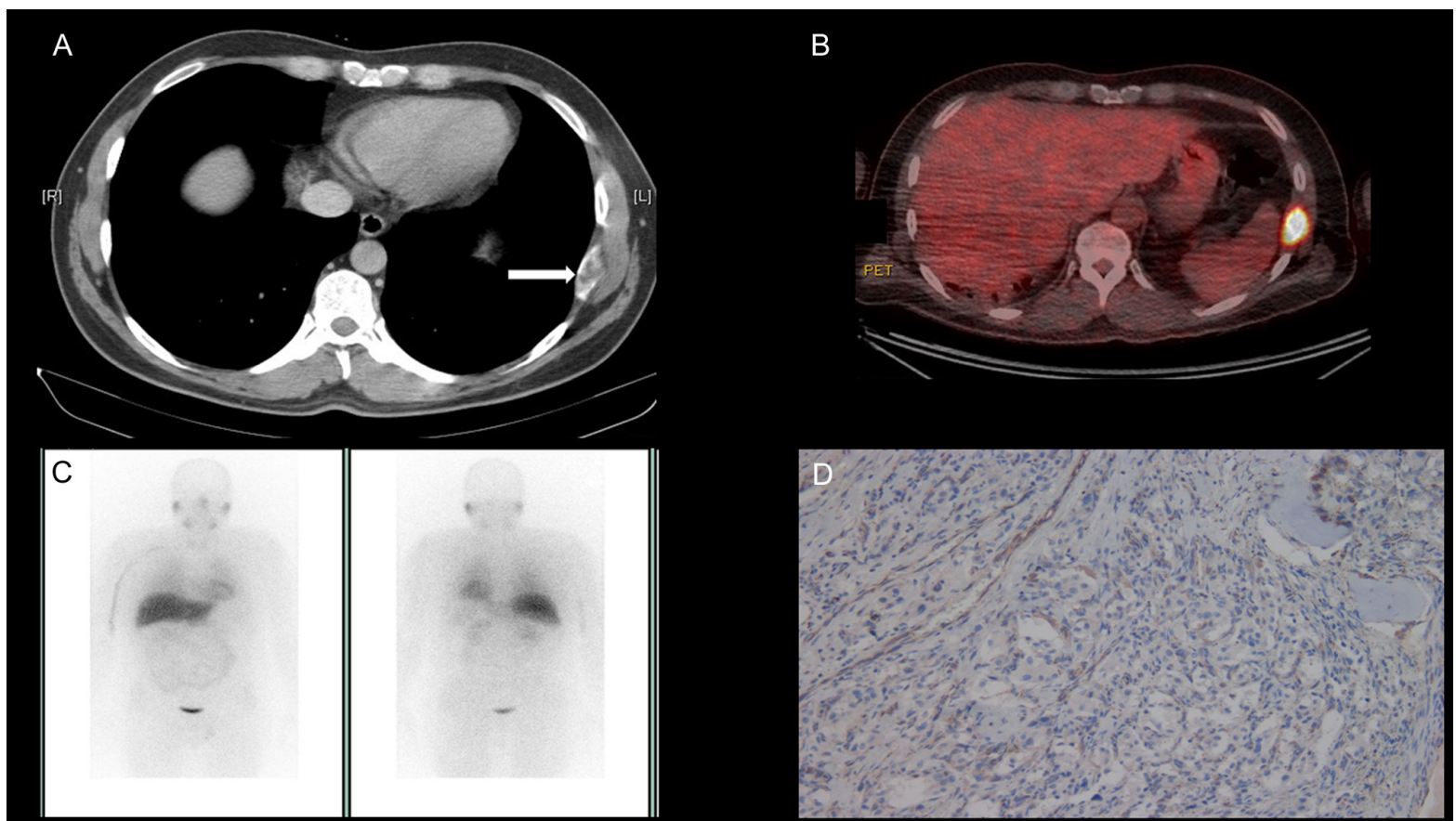

Figure 1

(A) Demonstrates the metastatic deposit in the left 8th rib on CT as indicated by the arrow. (B) Shows the avid rib metastasis on $18 \mathrm{~F}$ FDG PET CT and (C) shows no tumour avidity on 1131 MIBG imaging. (D) Demonstrates loss of SDHB protein expression in the metastatic tumour deposit on SDHB immunostaining indicating SDH deficiency due to the detected germline mutation in SDHA (c.91C > T, p.Arg31).

neck, thorax, abdomen and pelvis was performed and a metastatic deposit in the eighth left rib with no evidence of additional disease was identified (Fig. 1A). An ${ }^{131}$ I-MIBG scan was performed both for staging purposes and to evaluate suitability for ${ }^{131}$ I-MIBG therapy. Interestingly, the metastatic deposit was not avid (Fig. 1C). An ${ }^{18} \mathrm{~F}-\mathrm{FDG}$ PET CT confirmed avidity (maximum standard uptake value of 19.1) in the left rib lesion (Fig. 1B) but no additional disease was identified. The patient was referred for a thoracotomy and surgical resection of the 8th rib after appropriate preoperative optimisation with alpha-blockade. Subsequently, histological analysis confirmed the diagnosis of a metastatic paraganglioma with SDHB immunonegativity. SDHA immunohistochemistry was not performed (Fig. 1D).

Next generation sequencing of $S D H A, S D H B, S D H C$, SDHD, SDHAF2, MAX, TMEM127, VHL, RET and FH was performed on germline DNA from this patient after obtaining informed consent. The Illumina Trusight-One assay was used for sequencing and a mean coverage depth of $>20$ fold was achieved for $98 \%$ of the regions sequenced. Whole exon deletions, duplications and large rearrangements are not detected using this method and multiple ligation probe analysis was performed for VHL, SDHB, SDHC and SDHD. This testing identified a pathogenic truncating mutation in the SDHA gene (c.91C>T, p.Arg31*), which was confirmed by Sanger sequencing.
Post-operatively, repeat plasma metanephrine levels returned to normal (normetanephrine, $391 \mathrm{pmol} / \mathrm{L}$; metanephrine, $180 \mathrm{pmol} / \mathrm{L}$ ). This patient will remain under regular clinical follow-up for the development of synchronous or metachronous tumours. Given his germline SDHA mutation status and previous disease avidity on ${ }^{18 F-F D G ~ P E T ~ C T, ~ g o i n g ~ f o r w a r d, ~ t h i s ~ w i l l ~ b e ~ t h e ~ p r e f e r r e d ~}$ surveillance imaging modality if there is no detectable disease on conventional cross-sectional imaging using CT/ MRI, in the presence of elevated plasma metanpehrine or methoxytyramine levels.

In summary, the case we describe provides additional evidence for the risk of metastatic disease in $S D H A$ mutated PGL. Moreover, our report highlights the utility of ${ }^{18} \mathrm{~F}-\mathrm{FDG}$ PET CT in the detection of metastatic disease in patients with $S D H A$ mutations, as has previously been demonstrated in cases of $S D H B$ and $S D H D$ related tumours (Timmers et al. 2007). Interestingly, and with relevance to optimal surveillance strategies for patients with germline $S D H A$ mutations, the significant lag period (23 years) between initial presentation and the development of metastatic disease described in our case was also observed in two other cases of SDHA mutated malignant PGL (Tufton et al. 2017). The incidence of metastatic disease in $S D H B$ related PC and PGL has been reported in two studies as 19\% (Benn et al. 2006) and $16 \%$ (Srirangalingam et al. 2008) over a mean

Published by Bioscientifica Ltd. 
follow-up of 48 and 70 months, respectively. These data suggest that the development of metastatic disease may occur earlier in the disease course of $S D H B$-associated PGL/ PC. Further study is required to define the risk of malignant disease in SDHA-related PGL/PC and the median interval for the development of malignancy. Recent literature suggests that life-long surveillance, as recently recommended by the European Society of Endocrinology (Plouin et al. 2016), is crucial for patients with $S D H A$ gene mutations, in addition to those patients with mutations in other $S D H$ subunits (SDHB/ $S D H C / S D H D)$. However, we acknowledge that prospective studies with extended follow-up periods of 15-20 years may be necessary to reveal the true incidence of metastasis in SDHx related PGL and to stratify individual surveillance protocols for patients based on the SDHx germline subunit mutation.

Ruth T Casey ${ }^{1,2}$ Benjamin G Challis ${ }^{2}$

Alison Marker ${ }^{3}$

Deborah Pitfield ${ }^{2}$

Heok K Cheow ${ }^{4}$ Ashley Shaw ${ }^{4}$ Soo-Mi Park ${ }^{1}$ Helen L Simpson ${ }^{5}$ Eamonn R Maher ${ }^{1}$

${ }^{1}$ Department of Medical Genetics, University of Cambridge and NIHR Cambridge Biomedical Research Centre and Cancer Research UK Cambridge Centre, Cambridge, UK ${ }^{2}$ Department of Endocrinology, Cambridge University NHS Foundation Trust, Cambridge, UK ${ }^{3}$ Department of Histopathology, Cambridge University NHS Foundation Trust, Cambridge, UK ${ }^{4}$ Department of Radiology, Cambridge University Hospitals NHS Foundation Trust, Cambridge, UK ${ }^{5}$ Department of Diabetes and Endocrinology, University College London Hospitals, NHS Foundation Trust, London, UK

(Correspondence should be addressed to R Casey; email: rc674@medschl.cam.ac.uk)

Declaration of interest

The authors declare that there is no conflict of interest that could be perceived as prejudicing the impartiality of this article.

\section{Funding}

The authors thank the following funding agencies: NIHR (E R M), European Research Council Advanced Researcher Award (E R M), the British Heart Foundation (E R M) and Health Research Board Ireland (R C).

\section{References}

Bausch B, Schiavi F, Ni Y, Welander J, Patocs A, Ngeow J, Wellner U, Malinoc A, Taschin E, Barbon G, et al. 2017 Clinical characterization of the pheochromocytoma and paraganglioma susceptibility genes SDHA, TMEM127, MAX, and SDHAF2 for gene-informed prevention. JAMA Oncology [in press]. (doi:10.1001/ jamaoncol.2017.0223)

Benn DE, Gimenez-Roqueplo AP, Reilly JR, Bertherat J, Burgess J, Byth K, Croxson M, Dahia PL, Elston M, Gimm O, et al. 2006 Clinical presentation and penetrance of pheochromocytoma/paraganglioma syndromes. Journal of Clinical Endocrinology and Metabolism 91 827-836. (doi:10.1210/jc.2005-1862)

Boikos SA, Pappo AS, Killian JK, LaQuaglia MP, Weldon FC, Georger S, Trent JC, von Mehren M, Wright JA, Schiffman JD, et al. 2016 Molecular subtypes of KIT/PDGFRA Wild-type gastrointestinal stromal tumors: a report from the National Institutes Of Health Gastrointestinal Stromal Tumor Clinic. JAMA Oncology 2 922-928. (doi:10.1001/jamaoncol.2016.0256)

Burnichon N, Brière JJ, Libé R, Vescovo L, Rivière J, Tissier F, Jouanno E, Jeunemaitre X, Bénit P, Tzagoloff A, et al. 2010 SDHA is a tumor suppressor gene causing paraganglioma. Human Molecular Genetics 19 3011-3020. (doi:10.1093/hmg/ddq206)

Casey RT, Ascher DB, Rattenberry E, Izatt L, Andrews KA, Simpson HL, Challis B, Park S-M, Bulusu VR, Lalloo F, et al. 2017 SDHA related tumorigenesis: a new case series and literature review for variant interpretation and pathogenicity. Molecular Genetics and Genomic Medicine 5 237-250. (doi:10.1002/mgg3.279)

Evenepoel L, Papathomas TG, Krol N, Korpershoek E, de Krijger R, Persu A \& Dinjens WN 2015 Toward an improved definition of the genetic and tumor spectrum associated with SDH germ-line mutations. Genetics in Medicine 17 610-620. (doi:10.1038/ $\operatorname{gim} .2014 .162)$

Plouin PF, Amar L, Dekkers OM, Fassnacht M, Gimenez-Roqueplo AP, Lenders JW, Lussey-Lepoutre C, Steichen O \& Guideline Working Group 2016 European Society of Endocrinology Clinical Practice Guideline for long-term follow-up of patients operated on for a phaeochromocytoma or a paraganglioma. European Journal of Endocrinology 174 G1-G10. (doi:10.1530/EJE-16-0033)

Srirangalingam U, Walker L, Khoo B, MacDonald F, Gardner D, Wilkin TJ, Skelly RH, George E, Spooner D, Monson JP, et al. 2008 Clinical manifestations of familial paraganglioma and phaeochromocytomas in succinate dehydrogenase B (SDH-B) gene mutation carriers. Clinical Endocrinology 69 587-596. (doi:10.1111/j.1365-2265.2008.03274.x)

Timmers HJ, Kozupa A, Chen CC, Carrasquillo JA, Ling A, Eisenhofer G, Adams KT, Solis D, Lenders JW \& Pacak K 2007 Superiority of fluorodeoxyglucose positron emission tomography to other functional imaging techniques in the evaluation of metastatic SDHB-associated pheochromocytoma and paraganglioma. Journal of Clinical Oncology 25 2262-2269. (doi:10.1200/JCO.2006.09.6297)

Tufton N, Ghelani R, Srirangalingam U, Kumar VKA, Drake W, Iacovazzo D, Skordilis K, Berney DM, Al-Mrayat M, Khoo B, et al. 2017 SDHA mutated paragangliomas may be at high risk of metastasis. EndocrineRelated Cancer 24 L43-L49. (doi:10.1530/ERC-17-0030)

Received in final form 24 June 2017

Accepted 26 June 2017

Published by Bioscientifica Ltc. http://erc.endocrinology-journals.org DOI: $10.1530 /$ ERC-17-0206
() 2017 The authors Printed in Great Britain 